\title{
May a Relationship Occur Between Peripartum Cardiomyopathy and Restrictive Cardiomyopathy?
}

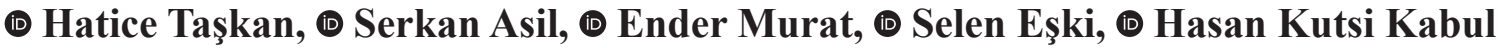

Gülhane Training and Research Hospital, Department of Cardiology, Ankara, Turkey

\begin{abstract}
Peripartum cardiomyopathy is a rare type of systolic heart failure at the end of pregnancy or in the months after birth, affecting young women. Restrictive cardiomyopathy is a rare form of predominantly diastolic heart failure and is difficult to diagnose. To date, there has been no case report showing the relationship between restrictive cardiomyopathy and peripartum cardiomyopathy. In this case report, a 35-year-old female patient who presented with heart failure symptoms and was diagnosed with restrictive cardiomyopathy and history of peripartum cardiomyopathy will be presented.
\end{abstract}

Keywords: Peripartum cardiomyopathy, restrictive cardiomyopathy, 'dip and plateau pattern' or 'square root sign'

\section{Introduction}

Restrictive cardiomyopathy (RCMP) is an uncommon category of the disease characterized by increased ventricular stiffness with poor prognosis and no effective treatment in the presence of normal diastolic volume and ventricular wall thickness ${ }^{(1,2)}$. Peripartum cardiomyopathy
(PPCM) is a form of systolic heart failure that affects young women at the end of pregnancy or in the months after birth ${ }^{(3)}$. To date, there has been no publication or case report on the relationship between these two clinical conditions. We will describe a novel case of RCMP with a history of PPCM that was 7 years ago.

Address for Correspondence: Serkan Asil, Gülhane Training and Research Hospital, Department of Cardiology, Ankara, Turkey e-mail: dr_serkanasil@hotmail.com ORCID: orcid.org/0000-0002-6782-4237

Received: 14.10.2021 Accepted: 12.11.2021

Cite this article as: Taşkan H, Asil S, Murat E, Eşki S, Kabul HK. May a Relationship Occur Between Peripartum Cardiomyopathy and Restrictive Cardiomyopathy? EJCM 2021;9(4):213-220.

DOI: 10.32596/ejcm.galenos.2021-10-051 


\section{Case Report}

A 35-year-old female was admitted to our clinic with the symptoms of leg swelling and shortness of breath. The patient's physical examination revealed rales in two lung bases and pretibial oedema, electrocardiogram showed incomplete right bundle branch block and loss of $\mathrm{R}$ progression in anterior leads. Complete blood count, kidney, liver function tests, and cardiac enzymes were found to be normal, PRO-BNP was $1185 \mathrm{mg} / \mathrm{dL}$.

The patient's medical history had PPCM. According to the patient's medical records of seven years ago, we obtained that left ventricular function decreased globally $(\mathrm{EF}=45 \%)$ in the transthoracic echocardiography and diastolic dysfunction was normal. The patient had been treated with ACE inhibitor and beta-blocker for 2 years after the diagnosis of PPCM. At the end of the $2^{\text {nd }}$ year, due to her clinical complete recovery, her medical treatment had been discontinued, and her follow-up echocardiograms showed normal systolic functions.

Transthoracic echocardiography performed on the patient's application to us showed that EF 45\%-50\% decreased left ventricular systolic functions in the midrange, dilated left and right atria, right ventricular systolic function was suppressed and increased in size. Doppler echocardiography showed increased early diastolic filling compared to atrial filling and increased $\mathrm{E} / \mathrm{A}$ and $\mathrm{E} / \mathrm{e}^{\prime}$ ratio (Figure 1). It was determined that there was moderate

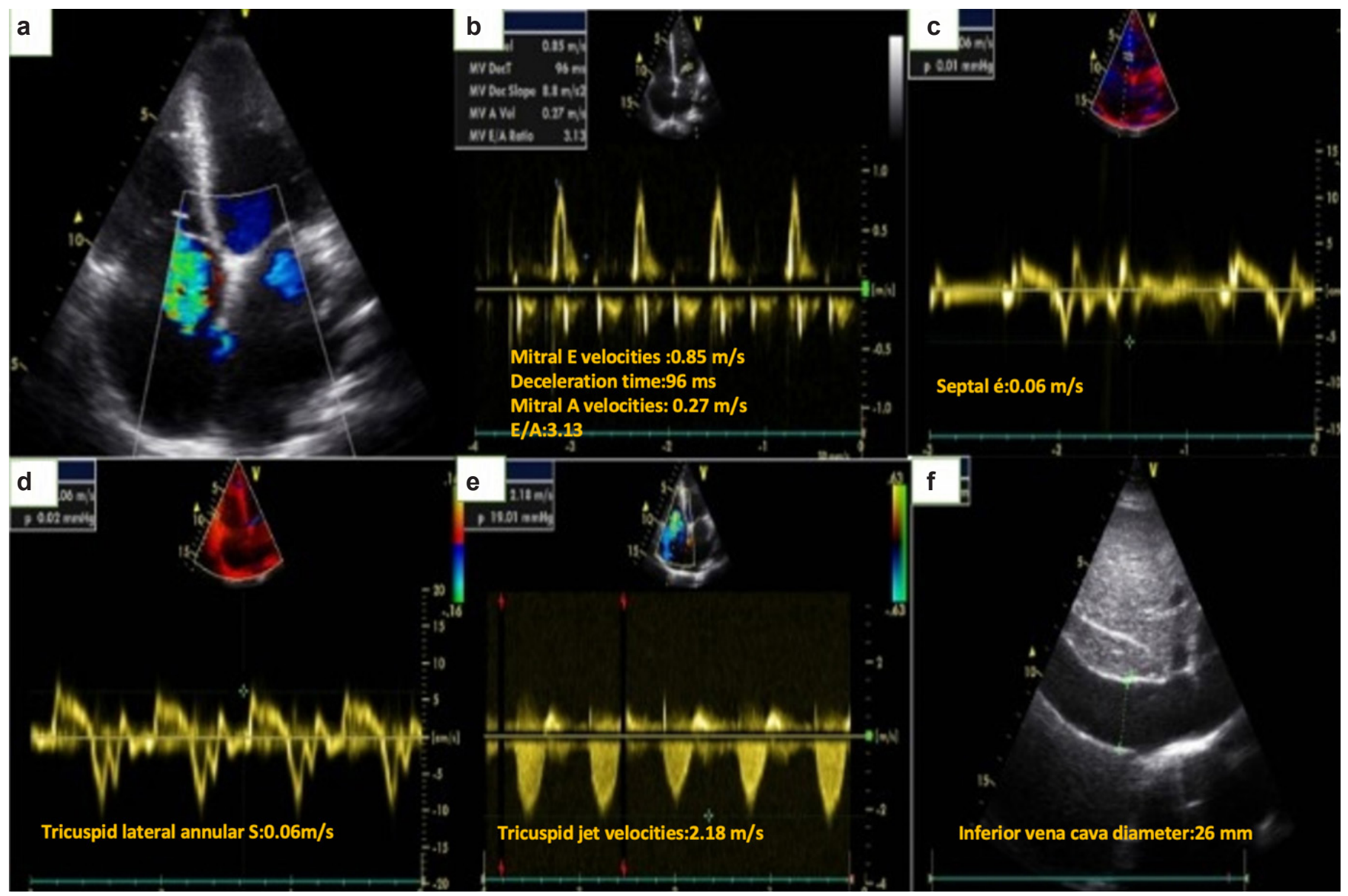

Figure 1. a) Echocardiography reveals biatrial enlargement. b) Doppler echocardiography shows increased early diastolic filling to the atrial filling ratio. c) Doppler echocardiography myocardial velocities of the basal septal wall. d) Tricuspid lateral annular S': $0.06 \mathrm{~m} / \mathrm{s}$. e) Severe tricuspid regurgitation, and tricuspid jet velocity: $2.18 \mathrm{~m} / \mathrm{s}$ f), inferior vena cava pleathore and measured diameter $26 \mathrm{~mm}$ 
mitral regurgitation, severe tricuspid regurgitation, and systolic pulmonary artery pressure of $40 \mathrm{mmHg}$. Tricuspid valve coaptation was impaired, passage from left to right through the patent foramen ovale (PFO) tunnel was observed (Figure 1). After that, we performed Cardiac magnetic resonance imaging (MRI) for differential diagnosis. Both ventricular functions decreased globally in cardiac MRI (Left ventricular ejection fraction was 36\%, right ventricular ejection fraction was $21 \%$ ). Late contrast images showed patchy mid-myocardial subepicardial areas of late enhancement in the left ventricular basis and mid-myocardial-subepicardial levels on all walls. No calcification, thickening or adhesion of the heart was detected in the pericardium (Figure 2).

Considering these findings, cardiac catheterization was performed on the patient with a pre-diagnosis of RCMP. Coronary arteries were found to be normal in coronary angiography. Left and right catheterization revealed that left ventricular pressure was $125 / 22 \mathrm{mmHg}$, aortic pressure was 125/72/90 $\mathrm{mmHg}$, right atrium pressure was $22 \mathrm{mmHg}$, right ventricular pressure was $40 / 22 \mathrm{mmHg}$, and mean pulmonary artery pressure was $28 \mathrm{mmHg}$. In the examination of the pressure records, it was observed that the diastolic pressure of both ventricles increased, and the difference between the diastolic pressure of the left and right ventricle was less than $5 \mathrm{mmHg}$. 'Dip and plateau pattern' or 'square root sign' was detected, and the diagnosis of RCMP was confirmed (Figure 2). The patient was evaluated as RCMP, and her medical treatment was arranged. In the follow-up, the implantable defibrillator was implanted in the patient who had progressive heart failure and non-sustained ventricular tachycardia attacks despite maximal medical therapy and was referred for heart transplantation list. After 10 months of follow-up, the patient, who was hospitalized with advanced heart failure and appropriate ICD shocks, died on the $3^{\text {rd }}$ day of his admission.

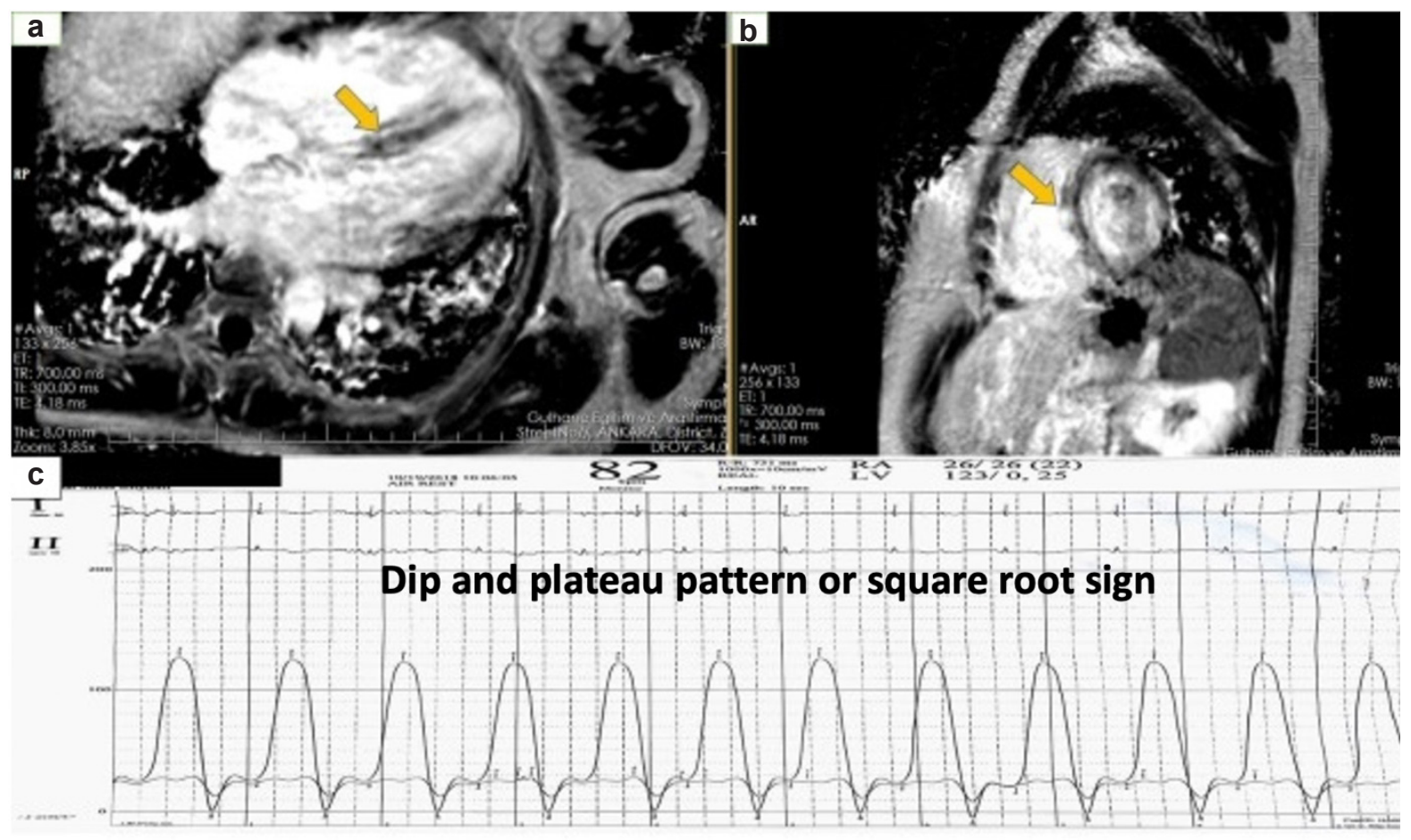

Figure 2. a, b) Cardiac magnetic resonance image of late enhancement in the left ventricular basis and midmyocardial-subepicardial levels on all walls. c) Equalization of left and right ventricular end-diastolic pressure and characteristic 'Dip and plateau pattern' or 'square root sign' 


\section{Discussion}

In this case report, it was emphasized that two very rare types of heart failure occurred in the same patient at different times. Although there were no clear data to reveal the relationship between PPCM and RCMP, this situation has created some suspicions. We wanted to share this case to draw attention that in the late period, PPCM may be involved in the pathogenesis of RCMP, and to define future cases.

RCMP can result from a combination of hereditary or acquired predispositions and diseases, which can be narrowly categorized as infiltrative, storage, non-infiltrative, and endomyocardial ${ }^{(4)}$. Certain echocardiographic and clinic signs and symptoms could raise the suspicion of specific diagnoses such as amyloidosis and desmopathies ${ }^{(5)}$. However, there were no such signs and symptoms suggestive of diagnoses in our patient. Increased creatine kinase is seen in desmopathies, and increased proteinuria is seen in amyloidosis ${ }^{(5)}$. In our case, CPK was normal and the patient had no proteinuria, no thickening of the left ventricular wall. Based on these results, we diagnosed our patient with idiopathic RCMP.

After we suspected that PPCM, which the patient had experienced during her pregnancy 7 years ago, might present with RCMP in the late period, we searched the patient's medical records and literature. However, we could not find any evidence of RCMP in our patient's medical record. Also, in the literature review, we could not find any evidence on that PPCM causes RCMP. There have been very few cases of pregnant RCMP patients ${ }^{(6,7)}$. So, indicating the presence of PPCM in the patient's history, we could not term the case as idiopathic RCMP. However, we think that the relationship between PPCM and RCMP in this patient will help to define future cases.

Patients with RCMP typically experience serious signs of heart failure within a brief span of time and, unless they undergo a cardiac transplant, most die within a few years after diagnosis ${ }^{(8)}$. In our case, the patient had an asymptomatic period of about 5 years after the diagnosis of peripartum cardiomyopathy, during which she did not receive any treatment.
In conclusion, should PCMP and RCMP be considered separate events in this case, or could PCMP have caused RCMP? Since both diseases are rare, they are much less likely to be seen in the same patient. This is a novel case of RCMP occurring seven years after PCMP. Although the underlying mechanism is not known, a relationship with PCMP is suspected.

\section{Ethics}

Informed Consent: It was obtained.

Peer-review: Externally peer-reviewed.

\section{Authorship Contributions}

Surgical and Medical Practices: S.A., Concept: S.A., H.K.K., Design: S.A., H.K.K., Data Collection and/or Processing: H.T., E.M., S.E., Literature Search: S.A., E.M., S.E., Writing: S.A., H.T.

Conflict of Interest: The authors declare that they have no conflict of interest.

Financial Disclosure: The authors declare no financial support by any grant or research sponsor and no competing financial interest.

\section{References}

1. Mogensen J, Arbustini E. Restrictive cardiomyopathy. Curr Opin Cardiol 2009;24:214-20

2. Hong JA, Kim MS, Cho MS, et al. Clinical features of idiopathic restrictive cardiomyopathy: A retrospective multicenter cohort study over 2 decades. Medicine (Baltimore) 2017;96:e7886.

3. Davis MB, Arany Z, McNamara DM, Goland S, Elkayam U. Peripartum Cardiomyopathy: JACC State-of-the-Art Review. J Am Coll Cardiol 2020 ;75:207-21.

4. Muchtar E, Blauwet LA, Gertz MA. Restrictive Cardiomyopathy: Genetics, Pathogenesis, Clinical Manifestations, Diagnosis, and Therapy. Circ Res 2017;121:819-37.

5. Rapezzi C, Arbustini E, Caforio AL, et al. Diagnostic work-up in cardiomyopathies: bridging the gap between clinical phenotypes and final diagnosis. A position statement from the ESC Working Group on Myocardial and Pericardial Diseases. Eur Heart J 2013;34:1448-58.

6. Nayak UA, Shekhar SP, Sundari N. A Rare Case of Pregnancy with Restrictive Cardiomyopathy. J Cardiovasc Echogr 2016;26:65-7.

7. Schaufelberger M. Cardiomyopathy and pregnancy. Heart 2019;105:154351

8. Benotti JR, Grossman W. Restrictive cardiomyopathy. Annu Rev Med $1984 ; 35: 113-25$ 